\title{
O ABORTO ENTRE ADOLESCENTES
}

\author{
Vera Lúcia Costa Souza* \\ Maria Suely Medeiros Corrêa** \\ Sinara de Lima Souza*** \\ Maria Aparecida Beserra****
}

Souza VLC, Corrêa MSM, Souza SL, Beserra MA. 0 aborto entre adolescentes. Rev Latino-am Enfermagem 2001 março; 9(2): $42-7$.

Para algumas adolescentes a gravidez significa realização e felicidade, mas para a maioria delas significa momento de tristeza, medo e até mesmo desespero, pois o filho não estava nos seus planos e o aborto se apresenta como única saída. Este estudo retrospectivo objetivou traçar o perfil epidemiológico das adolescentes internadas que se submeteram a curetagem, no Hospital Geral Clériston Andrade em Feira de Santana-Bahia, no período de janeiro/1995 a dezembro/1997. Os resultados demonstraram a necessidade de trabalho educativo como forma de prevenção da gravidez indesejada.

UNITERMOS: gravidez na adolescência

\section{THE ABORTION AMONG ADOLESCENTS}

For some adolescents, pregnancy means happiness and fullfillment. However, for most of them, it means a sad moment, characterized by fear and despair, as this is an unplanned situation and the abortion seems the only alternative. This retrospective study aimed at finding out the epidemiological profile of the adolescents admitted at the Clériston Andrade General Hospital, in Feira de Santana, Bahia, that were submitted to a curettage from January 1995 to December 1997. The results showed the need for educative programs in order to prevent unplanned pregnancies.

KEY WORDS: pregnancy in adolescence

\section{EL ABORTO ENTRE LOS ADOLESCENTES}

Para algunas adolescentes el embarazo felicidad y realización, pero para la mayoría de ellas significa un momento de tristeza, miedo e incluso desesperación, pues el hijo no estaba en sus planes y el aborto se presenta como única salida. Este estudio retrospectivo buscó trazar el perfil epidemiológico de las adolescentes hospitalizadas que fueron sometidas a legrado de enero del 1995 al diciembre del 1997. Los resultados señalaron una gran necesidad del trabajo educativo como alternativa para prevenir embarazos no deseados.

TÉRMINOS CLAVES: embarazo en la adolescencia

* Professor Assistente da Disciplina Enfermagem na Atenção à Saúde da Mulher, DSAU-Universidade Estadual de Feira de Santana, Mestranda do Programa de Pós-Graduação em Enfermagem- EEUFBA, área de concentração Enfermagem na Atenção à Saúde da Mulher, participante do GEM- Grupo de Estudos sobre Saúde da Mulher. Endereço: Rua: Coronel Ezequiel, 157 - Brasília - 44062-230 - Feira de Santana - Bahia - Brasil. E-mail: Sergvera@ gd.com.br

** Professor Auxiliar da Universidade de Pernambuco, Mestranda do Programa de Pós-Graduação em Enfermagem- EEUFBA, área de concentração Enfermagem na Atenção à Saúde da Mulher, participante do GEM- Grupo de Estudos sobre Saúde da Mulher. E-mail: Correa@ E-net.com.br

*** Professor Auxiliar da Disciplina Enfermagem na Atenção à Saúde da Criança e Adolescente DSAU-Universidade Estadual de Feira de Santana, Mestranda do Programa de Pós-Graduação em Enfermagem- EEUFBA, área de concentração Enfermagem na Atenção à Saúde da Criança e do Adolescente, participante do grupo CRESCER. E-mail: Sinara@gd.com.br

**** Professor Auxiliar da Universidade de Pernambuco, Mestranda do Programa de Pós-Graduação em Enfermagem- EEUFBA, área de concentração Enfermagem na Atenção à Saúde da Criança e do Adolescente, participante do grupo CRESCER 


\section{INTRODUÇÃO}

A fase de adolescência é caracterizada por etapas de desenvolvimento físico, mental, emocional e social, passando de uma fase de dependência socioeconômica total a outra de relativa independência. Sabe-se que a transição da infância para a fase adulta é um processo lento; no entanto, se uma adolescente engravida, esta fase é transposta aos saltos, quando ainda está se adaptando às transformações que estão ocorrendo em seu corpo.

A sociedade comporta-se de maneira contraditória, quando considera importante que 0 jovem exerça a liberdade sexual no momento que desejar e exalta a satisfação de todo ou qualquer desejo, porém a exaltação do prazer fica restrita às relações sexuais e o erotismo tem sido utilizado como meio de venda de produtos do prazer físico e a aquisição destes representa status social. Outras formas de prazer, que contribuiriam para o crescimento intelectual da adolescente, na maioria das vezes não são citadas. No entanto, nesta mesma sociedade, os valores modernos difundidos são os de percepção do amor em lugar da maternidade, como base de casamento, escolha individual e subjetiva do parceiro, maternidade retardada, estudo e trabalho fora do lar, que vem exigindo cada vez melhores qualificações. 0 aborto só é considerado útil enquanto a gravidez não se tornou pública. Desta maneira, a sociedade não oferece garantias para o exercício da sexualidade das adolescentes nem as adolescentes são conscientizadas das conseqüências que a sua liberdade sexual pode trazer, tendo tudo para se tornar traumática.

A sexualidade masculina e feminina construída socialmente cria estereótipos em que a sexualidade masculina é reconhecida como incontrolável, cheia de permissões e incentivos, e a sexualidade feminina é recheada de cobranças e restrições, devendo ser despertada e estar subordinada à vontade do homem. A ideologia responsabiliza a mulher e exime os homens de qualquer responsabilidade na questão da contracepção e da maternidade. A idéia que predominava e que ainda persiste é a responsabilização da mulher pela gravidez, mesmo nos casos de violência sexual ou risco de vida ${ }^{(1)}$.

A idade de iniciação das relações sexuais varia de um país para outro, segundo os valores regionais e culturais. No Brasil, $64 \%$ dos adolescentes do sexo masculino e $13 \%$ do sexo feminino de 15 a 17 anos são sexualmente ativos ${ }^{(2)}$. Desse modo, a atividade sexual dos adolescentes é um indicador da gravidez em idade precoce, fenômeno que está acontecendo em todos países, numa freqüência cada vez maior, segundo dados do Ministério da Saúde. Estimativas indicam que, no Brasil, cerca de 1.000 .000 de adolescentes engravidam todo ano, e 10,7\% terminam em aborto ${ }^{(3)}$. A queda ${ }^{(4)}$ progressiva da fecundidade no Brasil, através dos dados do Censo do FIBGE ${ }^{(5)}$, evidencia a diminuição do número de filhos, de 4,5 filhos por mulher em 1980, para 2,5 filhos. Porém, esta queda da fecundidade como fenômeno mundial é perceptível em todas as faixas etárias, exceto entre adolescentes ${ }^{(6)}$.

0 responsável por esta queda é o uso dos métodos contraceptivos, no qual a esterilização é responsável por $44 \%$, a pílula $41 \%$ ao lado do aborto ${ }^{(7)}$.

Pesquisa realizada em toda América Latina pelo Instituto Alan Guttmacher ${ }^{(8)}$ aponta que no Brasil uma taxa anual de 3,7 abortos para cada 100 mulheres, na faixa etária de 15 a 49 anos, e, no total de 4.693 .300 gestações ocorridas no país no ano de 1991, um total de 1.433 .350 terminaram em aborto, ou seja, $31 \%$ das gestações foram interrompidas.

Dados recentes apontam que entre 1993 e 1998, houve um aumento de $31 \%$ no percentual de parto entre meninas de 10 14 anos foram atendidas pela rede do SUS e que em 1998, 50 mil adolescentes submetidas à curetagem pós-aborto em hospitais públicos $^{(9)}$

0 aborto induzido ou provocado é um assunto conhecido de todos, um ato ilegal em nosso país, um caso típico de controvérsia quanto ao fundamento ético; um problema, porém, de saúde pública pela freqüência com que ocorre, sendo que no Brasil representa a quarta causa de morte materna, devido a complicações.

É uma prática comum em todas as classes sociais, idades e estado civil, porém, dependendo da situação financeira, os riscos são maiores ou menores, assinalando a diferença entre a adolescente de maior e de menor poder aquisitivo ${ }^{(3)}$. As adolescentes de maior poder aquisitivo utilizam as clínicas especializadas e têm acesso à assistência qualificada; enquanto, na maioria das vezes, as adolescentes pobres não recorrem ao aborto por não terem condições financeiras e, como alternativa, buscam pessoas não habilitadas e métodos abortivos rudimentares, que levam a graves complicações e morte. A clandestinidade transforma 0 aborto em um negócio lucrativo, garantindo a impunidade para aqueles que 0 realizam.

Os motivos que levam uma adolescente a engravidar são variados e de diversas ordens. Muitas pesquisas mostram que 0 inicio da atividade sexual pelos jovens é cada vez mais precoce; a transa faz parte do namoro, com baixa incidência do uso de métodos anticonceptivos. Umas desejam engravidar como parte do processo da busca da identidade. Porém, a desinformação é uma das principais causas, pois a falta de informação a respeito da sexualidade faz do assunto um tabu, e esta atitude provoca curiosidade, que muitas vezes é satisfeita entre amigos. Desse modo, as adolescentes engravidam sem ao menos saberem o que está acontecendo com seu corpo, por não associar a relação sexual com a fecundidade, por não tomarem medidas para prevenir uma gravidez. Só encaram 
o problema quando já estão grávidas e, para muitas delas, o aborto é um método contraceptivo.

Na adolescência a gravidez é sempre considerada de alto risco, porque pode propiciar o aparecimento de uma série de complicações para mãe e para o feto, pelas alterações biológicas, psicológicas, sociais e culturais que podem advir. Com relação às repercussões para a saúde da adolescente, a gravidez representa uma das principais causas de morte de mulheres entre 15 e os 19 anos seja por complicação na própria gravidez, no parto ou pela pratica clandestina do aborto ${ }^{(9)}$.

Para muitas adolescentes a gravidez pode significar realização e felicidade, fruto de um momento de prazer, sendo esta desejada. Porém, para maioria delas o resultado positivo de gravidez significa momento de tristeza, medo, insegurança e até mesmo desespero, pois a gravidez não estava nos seus planos e a responsabilidade pela maternidade recai totalmente sobre elas. A decisão de ser ou não ser mãe não é uma decisão fácil e o que, aparentemente, parece ser uma decisão individual, envolve uma série de fatores.

0 aborto torna-se, então, a única saída para estas adolescentes e, neste desafio, elas arriscam suas próprias vidas, quando decidem interromper a gravidez utilizando-se de quaisquer recursos que tenham à mão. Esta decisão muitas vezes é vivida de forma solitária e clandestina, ou sobre pressão dos parceiros ou familiares. 0 sentimento de abandono não significa necessariamente que sejam deixadas sozinhas, mas sim porque o parceiro e familiares são os primeiros a propor o aborto, sem maiores indagações ${ }^{(10)}$. Por ser proibido, o aborto leva a pressões psicológicas e sociais muito grandes, sendo carregado de medo, culpa, censura, vergonha, e estas adolescentes ainda enfrentam o desprezo, a humilhação e o julgamento dos profissionais de saúde.

0 presente trabalho tem por objetivo estudar as características epidemiológicas do aborto entre adolescentes internadas em um hospital geral de referência, submetidas à curetagem, no município de Feira de Santana, no período de janeiro/ 1995 a dezembro/1997.

\section{METODOLOGIA}

0 espaço empírico da pesquisa foi o Hospital Geral Clériston Andrade localizado no município de Feira de Santana-Bahia. É uma instituição pública estadual, de referência no município, que serve de campo de estágio para diversos cursos de nível superior e médio, e desenvolve curso de residência em medicina. Possui um Banco de Leite Humano, sendo considerado pelo UNICEF, "Hospital Amigo da criança". A citada instituição, na área da mulher, desenvolve ações de planejamento familiar, imunização, pré-natal, programa de controle e prevenção da menopausa e preventivo ginecológico e, no período de $1995 / 1996$, foi responsável por $21 \%$ do total das curetagens pós-aborto do Município, numa proporção de um aborto para cada 2 partos $^{(11)}$.

A coleta de dados foi realizada, no período de dois meses nos livros de ocorrência do centro obstétrico e nos prontuários das adolescentes que foram internadas no hospital. As variáveis foram categorizadas em demográficas (idade, estado civil), biológica (história obstétrica), sócio-econômicas (procedência, responsável, ocupação). Para o desenvolvimento da pesquisa foi encaminhada solicitação à direção do hospital, sendo realizada após consentimento. Os dados foram tratados através de freqüência absoluta e percentagem, apresentadas em tabelas.

\section{RESULTADOS}

Tabela 1 - Distribuição do total de ocorrência anual de parto e aborto, numa Instituição Pública de Feira de Santana-BA. Janeiro/1995 a Dezembro/1997

\begin{tabular}{|c|c|c|c|c|c|}
\hline \multirow[t]{2}{*}{ PERíodo } & \multicolumn{2}{|c|}{$\begin{array}{c}\text { OCORPÊHCL } \\
\text { DE PARTO }\end{array}$} & \multicolumn{2}{|c|}{$\begin{array}{c}\text { OCORPÊHCL } \\
\text { DEABORTO }\end{array}$} & \multirow[t]{2}{*}{$\begin{array}{c}\text { RELAV̄̃ñ } \\
\text { ABORTO/PARTO }\end{array}$} \\
\hline & $\boldsymbol{H}$ & $\mathbf{8}$ & $\boldsymbol{H}$ & $\mathbf{8}$ & \\
\hline Janeiro a Dezembro/1995 & 1679 & 499 & 907 & 47,5 & $1: 1,85$ \\
\hline Janero a Dezembro/1996 & 899 & 26,7 & 50 & 27,8 & $1: 1,69$ \\
\hline Janeiro a Dezembro/1997 & 789 & 23,4 & 472 & 24,7 & $1: 1,67$ \\
\hline 18 & 336 & 1000 & 1908 & 1000 & $1: 1,76$ \\
\hline
\end{tabular}

$\mathrm{Na}$ Tabela 1 verificou-se a queda no número geral de procedimentos obstétricos nos três anos consecutivos, porém o aborto apresenta uma queda menor em relação ao decréscimo verificado no número de partos. A relação aborto/parto em 1995 foi de 1/1,85 (um aborto para cada 1,85 partos), em 1996, esta relação foi de 1/1,7 partos e em 1997 de 1/1,7 partos.

Tabela 2 - Freqüência anual de aborto entre adolescentes, numa Instituição Pública de Feira de Santana-BA. Janeiro/1995 a Dezembro/1997
PEŔ́0DO

Janeiro a Dezembro/1995

Janeiro a Dezembro/1996

Janeiro a Dezembro/199?

TOWI

\section{FREQÜUEACLK DEABORTOERTRE} ADOLESCERTES

$\begin{array}{cc}\boldsymbol{H} & \text { \% } \\ 68 & 24,0 \\ 89 & 31,4 \\ 126 & 44,6 \\ \mathbf{2 8 3} & \mathbf{1 0 0 , 0}\end{array}$

Na Tabela 2, dos 283 casos de abortos entre as adolescentes, no período de janeiro/95 a dezembro/97, houve um 
aumento progressivo da ocorrência de aborto nos três anos consecutivos, passando de 24,0\% (1995), para 31,4\%(1996) e em 1997 para 44,6\%, apesar de ter ocorrido uma queda progressiva no número de ocorrência geral de abortos. Relacionado-se o número de aborto entre adolescentes com o total de ocorrência obstétrica anual, verificou-se que o aborto nesta faixa etária em 1995 representou 7,5\%, em 1996, 17,0\% e, em 1997, 27,0\%.

Tabela 3 - Distribuição por idade das adolescentes com aborto, internadas numa Instituição Pública de Feira de Santana-BA, Janeiro/ 1995 a Dezembro/1997

$\begin{array}{lcc}\text { IDNDE } & \boldsymbol{H} & \mathbf{\%} \\ 13 \text { anos } & 01 & 0,3 \\ 14 \text { anos } & 09 & 3,2 \\ 15 \text { anos } & 29 & 10,2 \\ 16 \text { anos } & 39 & 13,8 \\ 17 \text { anos } & 61 & 21,5 \\ 18 \text { anos } & 65 & 23,0 \\ 19 \text { anos } & 79 & 28,0 \\ \text { TOW } & \mathbf{2 8 3} & \mathbf{1 0 0 , 0}\end{array}$

Ao distribuir as adolescentes que abortaram por idade, 28,0\% apresentava 19 anos. Na faixa etária de 17 a 19 anos, a freqüência de aborto representou $72,5 \%$. Esta alta incidência de aborto entre as adolescentes traz preocupação, pois ${ }^{(12)}$, são grandes as repercussões negativas que estas adolescentes podem apresentar, por estarem no inicio de sua vida reprodutiva.

Tabela 4 - Estado civil das adolescentes com aborto internadas numa Instituição Pública de Feira de Santana -BA, Janeiro/1995 a Dezembro/1997

$\begin{array}{lcc}\text { ESWDO CNIL } & \boldsymbol{H} & \mathbf{\%} \\ \text { Solteiras } & 230 & 81,6 \\ \text { Casadas } & 34 & 12,0 \\ \text { União estável } & 19 & 6,4 \\ \text { TOWL } & \mathbf{2 8 3} & \mathbf{1 0 0 , 0}\end{array}$

Com relação à situação conjugal, 81,6\% das adolescentes que abortaram eram solteiras, sugerindo que a grande maioria destas jovens, sem companheiros, decidiu abortar. Os dados confirmam resultados encontrados por outros pesquisadores, de que a maioria das mulheres que abortam $70 \%$, são solteiras ${ }^{(13)}$, e também foi encontrada uma taxa de $57,5 \%$ de aborto entre adolescentes solteiras ${ }^{(4)}$. A qualidade do relacionamento da adolescente com o parceiro é fundamental na opção de manter ou não a gravidez ${ }^{(14)}$. No momento em que o parceiro recusa-se a assumir a paternidade ou há incerteza dos sentimentos da jovem em relação ao parceiro mesmo quando este se dispõe a assumir a paternidade ou união, a adolescente se decide pelo aborto. Geralmente o parceiro é também adolescente ou adulto jovem, sem estrutura emocional e financeira para começar uma família ${ }^{(4)}$.

Quanto à ocupação, 68,0\% das adolescentes foram registradas nos prontuários como domésticas, e apenas $26,4 \%$ como estudantes. Os prontuários não especificam se estas adolescentes eram donas-de-casa, empregadas domésticas, ou foram consideradas domésticas por não ter sido identificada nenhuma ocupação. Apenas 5,6\% desenvolvem atividades remuneradas, enquanto 94,6 destas jovens sem nenhuma renda dependem financeiramente da família ou do companheiro. Sabe-se que as jovens com menos de quatro anos de estudo tendem a casar, ou engravidar mais cedo, como também a própria gravidez leva as jovens a abandonar os estudos, diminuindo assim as suas perspectivas de ascensão social.

Quanto à procedência, 79,2\% das adolescentes residem em Feira de Santana e 20,8\% são de outros municípios baiano. Algumas destas jovens que procederam de outros municípios, vieram transferidas de hospitais que não possuíam condições de atendimento, como também outras procuraram o hospital em Feira de Santana como forma de manter em sigilo a prática abortiva.

Tabela 5 - Distribuição da história obstétrica das adolescentes internadas numa Instituição Pública de Feira de Santana-BA, Janeiro/ 1995 a Dezembro 1997

\begin{tabular}{|c|c|c|c|c|c|c|c|c|}
\hline \multirow{2}{*}{$\begin{array}{l}\text { OCORPÊHCK } \\
\text { OBSTÉTRICA }\end{array}$} & \multicolumn{2}{|c|}{1} & \multicolumn{2}{|c|}{2} & \multicolumn{2}{|c|}{$>2$} & \multicolumn{2}{|c|}{ TOWL } \\
\hline & H & $\$$ & $\boldsymbol{H}$ & \% & $\boldsymbol{H}$ & $\$$ & $\boldsymbol{H}$ & $\mathbf{\Phi}$ \\
\hline$N^{0}$ de Gestação & 136 & 48,0 & 97 & 34,4 & 50 & 17,6 & 283 & 100,0 \\
\hline$N^{0}$ de Áborto & 272 & 96,0 & 11 & 4,0 & - & - & 283 & 100,0 \\
\hline
\end{tabular}

Entre as jovens, 48,0\% abortaram em sua primeira gestação, enquanto 52,0\% apresentam história de duas gestações ou mais, confirmando ${ }^{(3)}$ a probabilidade de essas adolescentes engravidarem novamente, após 36 meses da primeira gestação. Neste grupo estudado, 96,0\% teve o primeiro aborto. Isto mostra a necessidade de intervenção educativa sobre a importância do Planejamento Familiar, os riscos da gravidez na adolescência, esclarecimento sobre as complicações a que estão sujeitas com a prática abortiva, a fim de evitar a repetição do aborto, destacando haver maior probabilidade da ocorrência de novo aborto ${ }^{(15)}$, por serem jovens, estarem no início de sua vida reprodutiva, e não usarem regularmente algum método contraceptivo. 
Tabela 6 - Distribuição do tipo de aborto, numa Instituição Pública de Feira de Santana-Ba, Janeiro/1995 a Dezembro/1997

\section{TIPO DEABORTO}

Aborto Provocado

iborto lgnorado

uborto Espontâneo

TOW

0 aborto provocado representou $55,2 \%$ do total das ocorrências, seguido do aborto ignorado em $30,4 \%$, que neste trabalho foi considerado 0 aborto no qual não foi registrado a natureza em prontuário. 0 aborto ignorado pode ser considerado como provocado, pois pelo constrangimento do ato ilegal, a adolescente muitas vezes omite ou nega a utilização de método abortivo. Assim como, estes registros dependem da atitude do profissional frente ao aborto. Estes dados diferem dos encontrados $^{(16)}$, que foi de $37,3 \%$ de aborto provocado entre adolescentes. 0 aborto espontâneo foi mais freqüente entre adolescentes na faixa etária de 13 a 15 anos ${ }^{(17)}$, devido à imaturidade do aparelho reprodutivo podendo ocorrer a dilatação progressiva e amolecimento do colo uterino.

Segundo a idade gestacional, o aborto ocorreu com maior freqüência entre o segundo $(46,0 \%)$ e o terceiro $(33,0 \%)$ mês de gestação. Outro estudo ${ }^{(16)}$ mostrou $45,9 \%$ de aborto no segundo mês, $22,9 \%$ no terceiro mês e $23,1 \%$ no quarto mês. 0 baixo percentual no primeiro mês pode indicar que estas jovens, por desconhecimento da fisiologia do seu próprio corpo, não reconhecem a amenorréia como possível gravidez, mas este pode ser o momento de negociação com o parceiro.

Tabela 7 - Acompanhante da adolescente no momento do internamento, numa Instituição Pública de Feira de Santana-BA, Janeiro/1995 a Dezembro/1997

\begin{tabular}{|c|c|c|}
\hline MCOHPAHHАHE & $\boldsymbol{H}$ & \% \\
\hline Mãe & 90 & 32,0 \\
\hline Ámiga & 72 & 25,6 \\
\hline Outros parentes & 68 & 24,0 \\
\hline Parceiro & 30 & 10,4 \\
\hline Sozinha & 23 & 8,0 \\
\hline TOWL & 283 & 100,0 \\
\hline
\end{tabular}

Ao analisar a participação de terceiros no acompanhamento das adolescentes no momento do internamento, verificou-se que a mãe esteve presente em 32,0\% dos casos, seguido da amiga (25,6\%), outros parentes em $24,0 \%$ dos casos (representado por tia, irmã, prima, sogra), o parceiro acompanhoua em $10,4 \%$ e $8,0 \%$ das adolescentes procuraram o hospital sozinhas. Este dado mostra que a adolescente necessita do apoio de terceiros tanto na decisão de abortar, como no encaminhamento ao hospital para tratamento.

Das 283 adolescentes que abortaram, 243 não apresentaram complicações imediatas $(85,9 \%)$, permanecendo internadas apenas 24 horas, enquanto 40 adolescentes apresentaram complicações (14,1\%). Dentre as quais $57,5 \%$ infecção e 42,5\% hemorragia. A complicação infecciosa está relacionada à demora na procura de assistência médica, às mulheres solteiras (por falta de apoio do parceiro) e a mulheres de condição socioeconômica baixa, por dificuldade de acesso aos serviços de saúde ${ }^{(18)}$. Já as complicações hemorrágicas estão relacionadas à idade gestacional, ou seja, quanto maior for a idade gestacional, maior a incidência desta complicação.

\section{CONSIDERAÇÕES FINAIS}

Frente aos dados apresentados pode-se verificar que: a maior freqüência de abor to foi entre adolescentes de 17 a 19 anos, solteiras (81,6\%), em que 147 adolescentes abortaram após a primeira gravidez (52\%) e no segundo mês de gestação $(46,7 \%)$; 0 aborto provocado, bem como 0 aborto ignorado foram mais freqüentes, $55,2 \%$ e $30,4 \%$ respectivamente; dentre as complicações constatadas, o aborto infectado representou o maior percentual $(57,5 \%)$.

Medidas preventivas poderão evitar a repetição da prática abortiva ou postergar uma próxima gravidez, visto que a grande maioria das adolescentes investigadas realizou o aborto pela primeira vez. 0 trabalho educativo prévio e durante o internamento, o encaminhamento para o serviço de Planejamento Familiar, assim como a realização de campanhas de esclarecimentos sobre sexualidade, contracepção, os riscos e as complicações a que estão sujeitas ao se submeterem ao aborto torna-se de fundamental importância para uma vida sexual segura.

Além disso, o tratamento humanizado pelos profissionais de saúde é imprescindível devendo ser demonstrado através do respeito a opção da adolescente pelo aborto, considerando o período de internamento como oportunidade de escuta da adolescente sobre os fatores psicossociais que envolvem esta decisão, e promover o conhecimento com base na troca de saberes e não com imposição. 


\section{REFERÊNCIAS BIBLIOGRÁFICAS}

1. Araújo MJ. Implantação dos serviços legais na perspectivas dos direitos reprodutivos. In: Batista C, Laranjeira M, organizadores. Aborto: desafios da legalidade. Recife: SOS CORPO; 1998.

2. Henriques-Mueller MH, Yunes J. Adolescencia: equivocaciones y esperanzas. In: OPAS. Genero, mujer y salud: en las Americas. Washington: OPAS; 1993.

3. Takiuti AD. A saúde da mulher adolescente-1993. In: Madeira FR. Quem mandou nascer mulher? Rio de Janeiro: Record/Rosa dos Tempos; 1997. p. 213-90.

4. Bruno, ZV. Abortamento: aspectos epidemiológicos. Femina 1993; 9(21): 912-6.

5. Fundação IBGE. Censo demográfico, n. 17 - Bahia. Rio de Janeiro: IBGE; 1991.

6. Ministério da Saúde. Secretaria de Assistência à Saúde. DAPS. Coordenação Materno-Infantil-COMIN. Serviço de Assistência à Saúde do Adolescente-SASAD (BR). Normas de atenção integral do adolescente: assistência ao pré-natal, ao parto e ao puerpério, planejamento familiar, doenças sexualmente transmissíveis, problemas ginecológicos. Brasília: MS; 1993.

7. Berquó E, Araújo MJO, Sorrentino SR. A transição da fecundidade e o custo em saúde por parte das mulheres: o caso do Brasil. São Paulo: CEBRAP/FNUAP; 1993.

8. Institute Alan Guttmacher. Aborto clandestino: uma realidade latinoamericana. Nova lorque: Alan Guttmacher Institute; 1994.
9. Ministério da Saúde. Secretaria de Políticas de Saúde (BR). Saúde e desenvolvimento da juventude brasileira: construindo uma agenda nacional. Brasília: MS; 1999.

10. Chauí, M. Mãe, mulher ou pessoa: discutindo aborto. São Paulo: Lua Nova; s.d.

11. Souza VLC. Aborto: a problemática em Feira de Santana. Sitientibus jul./dez. 1998; 1(19): 9-25.

12. Costa CFF, Costa HLFF, Machado KM, Melo MPV, Rocha YKW, Costa LOBF. Aspectos epidemiológicos comparativos entre abortamento provocado e espontâneo. Rev Bras Ginecol Obstet 1996; 18(2): 179-84.

13. Boehs AE, Santos EK, Hasse M, Souza ML. Aborto provocado: estudo epidemiológico descritivo numa maternidade de Florianópolis - Santa Catarina. Ci Cult 1983; 4(35): 501-6.

14. Desser NA. Adolescência: sexualidade e culpa. Rio de Janeiro: Rosa dos Tempos; 1993.

15. Fagundes A, Cecatti JG. Morte materna: uma tragédia evitável. $2^{\mathrm{a}}$ ed. São Paulo: Editora da Unicamp; 1991.

16. Costa CF, Santos RR, Maia VA, Costa EL, Alencar AV. Abortamento: epidemiologia. Rev Bras Ginecol Obstet 1993; 5(5): 217-20.

17. Strasburger VC. Ginecologia básica da adolescente: guia para consultório. São Paulo: Santos; 1992.

18. Gabiatti JRE, Pinto Neto AM, Costa-Paiva LHS, Torres JCC. Fatores associados às complicações do abortamento. Rev Bras Ginecol Obstet 1995; 17(5): 537-42. 